\title{
Nonlinear dynamics of discrete time multi-level leader-follower games
}

\author{
Ruijia Wu and Robert A. Van Gorder* \\ Mathematical Institute, University of Oxford, Andrew Wiles Building, Radcliffe Observatory Quarter, Woodstock Road, Oxford OX2 $6 G G$ UK \\ *Robert.VanGorder@maths.ox.ac.uk
}

\begin{abstract}
We study dynamic multiple-player multiple-level discrete time leader-follower games in the vein of Cournot or Stackelberg games; these games generalize two-player dynamic Stackelberg or Cournot duopoly games which have been considered recently. A given player acts as a leader toward players in lower levels, and as a follower toward players in higher levels. We consider the case of either perfect or incomplete information, which in this context means that players either have complete information about other players within their level (perfect information) or lack information at the present timestep about other players within their level (incomplete information). Players always have perfect information about all players which are (relative) followers, and incomplete information about players which are (relative) leaders. The Cournot-type adjustment process under these information structures at each timestep results in the temporal dynamics of the game. As we consider dynamic games, we observe a variety of behaviors in time, including convergence to steady state or equilibrium quantities, cycles or periodic oscillations, and chaotic dynamics. We find that the costs facing each player strongly influence the form of the long-time dynamics, as will the information structure (perfect or incomplete) selected. One interesting finding is that under perfect information players tend to quickly converge upon their respective equilibrium values, while incomplete information can result in loss of regularity and the emergence of periodic or chaotic dynamics. However, in cases where players may be pushed out of the game in the presence of high relative costs and perfect information, we find that non-equilibrium dynamics under incomplete information allow such players to retain positive production, hence they are able to remain in the game.
\end{abstract}

Keywords: leader-follower games, dynamic games, information hierarchy, nonlinear dynamics

\section{Introduction}

There have been a number of studies on Cournot games for two (duopoloy) or more players, phrased as dynamic games on discrete or continuous time domains (Agizaand and Elsadany, 2003; Agliari et al., 2000; Askar and Alnowibet, 2016; Elsadany et al., 2013; Puu, 1991a,b; Puu, 1998; Puu, 2008; Snyder et al., 2013).

In Cournot games, all players have equal information and make moves simultaneously. However, real-life asymmetry in such dynamics has motivated the consideration of other models. In particular, one direction one can take is to consider leader-follower models, where a leader has more information on the state of the game than does a follower. A classical example of such a game would be a Stackelberg game (Von Stackelberg, 1934) which is a type of leader-follower game. In the Stackelberg framework, two players no longer decide their outputs independently yet simultaneously. Instead, the leader decides their move first, given knowledge of the situation at the previous timestep (Sherali et al., 1983). When the leader knows the reaction function of the follower, which depends on the output of leader, then it can determine its own output by maximizing its profit function. After the leader's decision is implemented, the follower has to produce the amount that has already been determined by the leader due to the reaction function. Since in a leader-follower model the leader is able to obtain the response of the followers given any policy made by the leaders, the leader is able to obtain the best output and reach their equilibrium in one iteration. Dynamic Stackelberg games have been considered, with a variety of dynamics (including periodic and chaotic behaviors) observed; see (Peng and Lu, 2015; Peng et al., 2016; Puu, 1998; Tramontana et al., 2011). It is possible to extend such models to multiple players which may take the role of either leaders or followers (see, for instance, Sherali et al. 1984; Van Gorder and Caputo, 2010). In addition to classical economic applications, such dynamic leader-follower games 
have seen recent application in areas such as communications networks (Xu et al., 2010), signaling games (Ahmed and Hegazi, 2006), and service-for-prestige theory (Price and Van Vugt, 2014).

Stackelberg strategies were considered for multilevel systems by Cruz (1976). Such concepts are useful in the multilevel hierarchical control problem of multicontroller distributed-parameter systems (Cruz, 1978; Tzafestas, 1980). Nie (2007) considered discrete-time dynamic multi-leader-follower games with the assumption of feedback perfect information, which extended the static multi-leader-follower games of Pang and Fukushima (2005). Applications of multi-level or hierarchical games and related systems include internet pricing (Shen and Basar, 2004), information theory (DAmato et al., 2012), incentives (Li et al., 2002), and cellular network deployment (Guruacharya et al., 2013).

While discrete and continuous time dynamics have been considered in the past 20 years or so for variations of the two- or many-player dynamic Cournot games (Agizaand and Elsadany, 2003; Agliari et al., 2000; Askar and Alnowibet, 2016; Elsadany et al., 2013; Puu, 1998; Puu, 2008; Snyder et al., 2013), and dynamic Stackelberg games and related variations on leader-follower games (Peng and Lu, 2015; Peng et al., 2016; Puu, 1998; Tramontana et al., 2011), the corresponding nonlinear dynamics arising from multi-level leader-follower games has not been wellstudied. In the present paper, we shall consider two-level and then multi-level leader-follower dynamic games in discrete time. As expected, the dynamics depend not only on the parameter values, but also on the information structure adopted, with a range of dynamics from stable equilibrium, to limit cycles and periodic orbits, and then chaos, being found. Indeed, there are interesting findings which do not seem to be studied in two player leaderfollower games, or games with only two-tier hierarchies.

Game theory has of course seen application to economic problems such as Cournot adjustment processes involving competing firms. However, there are a variety of other applications for game theory in the dynamic setting which may be considered. Such research could be of relevance for effective vaccination strategies (Wang et al., 2016), for understanding human cooperation (Perc, 2016) and collusion (Wernerfelt, 1989) or more general human behavior (Rassenti et al., 2000), to combat crime (D'Orsogna and Perc, 2015), standards of quality for products or services (Valletti, 2000), or even for saving human lives in general (Helbing et al., 2015). Hence, a better understanding of the dynamics of such games can be of benefit outside of economics, as well.

The paper is organized as follows. Section 2 reviews useful aspects of the dynamic Cournot games. In Section 3, we introduce generalizations of duopoly games to two-level (a leader level and a follower level), with each level having some positive integer number of players. We discuss the role of incomplete information versus perfect information within the leader level, and show that incomplete information may result in a loss of regularity and the emergence of non-equilibrium dynamics, such as periodic oscillations and chaos. In Section 4, we extend the model further, to consider multiple levels, with players in each level acting as leaders to those in levels below, and as followers to those in levels above. Again, we find that information structure influences the dynamics observed. Finally, we summarize and discuss the results in Section 5.

\section{Review of Cournot Model Results}

In this section we shall review material on Cournot dynamic games, as this will help to motivate the more general models which follow in Sections 3 and 4. The results here motivate the need to consider information asymmetry. For related differential games related to control theory, leader-follower games with multiple-players have been discussed in several contexts. Optimal control approaches for the dynamic games are discussed in Van Gorder and Caputo (2010) (and references therein). Multiple-level leader-follower games which are Stackelberg-like in nature have been studied by Cruz (1978). Gardner and Cruz (1978) considered feedback Stackelberg games for the multi-level or hierarchical setting, while the closed-loop control was studied by Basar and Olsder (1980). A control problem for another class of hierarchical leader-follower games was considered by Pan and Yong (1991).

Two frequently used functional forms for price in terms of quantity are $P=A-Q$ or $P=A / Q$ (Tramontana et al., 2011) where $A$ is a constant and $Q$ is the total output quantity. Sometimes, a variable can be added to represent government intervention such as taxation or subsidy (Snyder et al., 2013). We can also set a upper bound for the price to avoid infinite price as $Q$ tends to zero for $P=\frac{1}{Q}$ (Snyder et al., 2013). Since we find that players with linear price functions always approach fixed equilibrium values in finite time, this paper focuses on the inversely proportional price function $P=A / Q$. This function has been employed in many of the aforementioned studies on Cournot or Stackelberg games, and hence maintains relevance to possible applications. 
The Cournot model describes a market where all the players in the market are independent decision makers. Each player only knows the previous outputs of the other players, $q_{j}(t)$, and will try to maximize its profit at the next time step

$$
\Pi_{i}(t+1)=q_{i}(t+1) P\left(\mathbf{q}_{\mathbf{j}: \mathbf{j} \neq \mathbf{i}}(t), q_{i}(t+1)\right)-c_{i} q_{i}(t+1)
$$

where $P$ is the price function which depends on the total output, $q_{i}(t+1)$ is the output of the $i$ th player at time $t+1$, $\mathbf{q}_{\mathbf{j}: \mathbf{j} \neq \mathbf{i}}(t)$ represents the vector of outputs for all the other players, and $c_{i}$ is the marginal cost faced by the $i$ th player.

The profit (1) is maximized when $q_{i}(t+1)$ satisfies

$$
P\left(\mathbf{q}_{\mathbf{j}: \mathbf{j} \neq \mathbf{i}}(t), q_{i}(t+1)\right)+q_{i}(t+1) P^{\prime}\left(\mathbf{q}_{\mathbf{j}: \mathbf{j} \neq \mathbf{i}}(t), q_{i}(t+1)\right)-c_{i}=0 .
$$

Since $\mathbf{q}_{\mathbf{j}: \mathbf{j} \neq \mathbf{i}}(t)$ are already known, we may in principle solve (2) for $q_{1}(t+1)$, to obtain

$$
q_{i}(t+1)=R_{i}\left(\mathbf{q}_{\mathbf{j}: \mathbf{j} \neq \mathbf{i}}(t)\right),
$$

for the $i$ th player. This gives a system of $n$ difference equations for the dynamics of the $n$-player game. The system has equilibrium points $\left(q_{1}^{*}, \ldots, q_{n}^{*}\right)$ provided that $q_{i}(t)=q_{i}^{*}$, with $q_{i}^{*}$ a constant, for each $1 \leq i \leq n$.

As an example, assume there are two players $(n=2)$. Let $P\left(q_{1}, q_{2}\right)=\frac{1}{q_{1}+q_{2}}$. The profit functions (1) become

$$
\begin{aligned}
& \Pi_{1}(t+1)=q_{1}(t+1) \frac{1}{q_{1}(t+1)+q_{2}(t)}-c_{1} q_{1}(t+1), \\
& \Pi_{2}(t+1)=q_{2}(t+1) \frac{1}{q_{1}(t)+q_{2}(t+1)}-c_{2} q_{2}(t+1) .
\end{aligned}
$$

Setting $\frac{\partial \Pi_{1}}{\partial q_{1}}=0$ and $\frac{\partial \Pi_{2}}{\partial q_{2}}=0$, and solving for $q_{1}$ and $q_{2}$, we obtain the system of difference equations

$$
\begin{aligned}
& q_{1}(t+1)=\sqrt{\frac{q_{2}(t)}{c_{1}}}-q_{2}(t), \\
& q_{2}(t+1)=\sqrt{\frac{q_{1}(t)}{c_{2}}}-q_{1}(t) .
\end{aligned}
$$

We may simulate this system forward in time, in order to determine the dynamics of each player. We find that the positive equilibrium values are

$$
\begin{aligned}
& q_{1}^{*}=\frac{c_{2}}{\left(c_{1}+c_{2}\right)^{2}}, \\
& q_{2}^{*}=\frac{c_{1}}{\left(c_{1}+c_{2}\right)^{2}} .
\end{aligned}
$$

Note that $q_{1}^{*}=0$ and $q_{2}^{*}=0$ is the other possible equilibrium, and that asymmetric equilibrium values (with only one of $q_{i}^{*}=0$ ) are not possible.

\section{Two-Level Games With Multiple Leaders And Multiple Followers}

In this section, the case of several leaders and several followers in the market are considered. Such a model will hold two-player Stackelberg leader-follower games as a special case. Here the followers will take the leader outputs as given at the previous time step, and then update their dynamics accordingly. The leader will know the follower outputs in the present time, and update according to this and their own previous outputs. This is in contrast to the Cournot game in discrete time, where each player updates output given their own and the other players' outputs at the previous time step. This asymmetric adjustment process is what drives the dynamics of the game.

We consider two possibilities related to information. Firstly, we can assume leaders are independent decision makers and followers decide their outputs independently when the leaders' outputs are known, which means between the leaders and the followers, the leader-follower model is applied, and within the groups of leaders and followers, the Cournot model is applied. In this case, leaders have incomplete information as they do not know the current movements of other leaders. The second case, leaders all have perfect information of other players including other leaders. Either way, all leaders are of a similar tier, so we can consider these two-level games.

We now consider a game with $M \geq 1$ leaders and $N \geq 1$ followers. We shall consider both types of information mentioned above. 


\subsection{Leaders With Incomplete Information}

The system consists of $M \geq 1$ leaders and $N \geq 1$ followers with leaders having incomplete information on other leaders. The profit functions for the leaders and followers are as follows:

$$
\begin{aligned}
& \Pi_{i}^{L}(t+1)=\frac{A q_{i}^{L}(t+1)}{q_{i}^{L}(t+1)+\sum_{\substack{j \neq i \\
j=1}}^{M} q_{j}^{L}(t)+\sum_{j=1}^{N} q_{j}^{F}(t+1)}-c_{i}^{L} q_{i}^{L}(t+1), \\
& \Pi_{i}^{F}(t+1)=\frac{A q_{i}^{F}(t+1)}{q_{i}^{F}(t+1)+\sum_{j=1}^{M} q_{j}^{L}(t)+\sum_{\substack{j \neq i \\
j=1}}^{N} q_{j}^{F}(t)}-c_{i}^{F} q_{i}^{F}(t+1) .
\end{aligned}
$$

The optimality conditions then imply that the output quantities evolve in discrete time as

$$
\begin{aligned}
& q_{i}^{F}(t+1)=\sqrt{\frac{A}{c_{i}^{F}}\left(\sum_{j=1}^{M} q_{j}^{L}(t)+\sum_{\substack{j \neq i \\
j=1}}^{N} q_{j}^{F}(t)\right)}-\sum_{j=1}^{M} q_{j}^{L}(t)-\sum_{\substack{j \neq i \\
j=1}}^{N} q_{j}^{F}(t), \\
& q_{i}^{L}(t+1)=\sqrt{\frac{A}{c_{i}^{L}}\left(\sum_{\substack{j \neq i \\
j=1}}^{M} q_{j}^{L}(t)+\sum_{j=1}^{N} q_{j}^{F}(t+1)\right)}-\sum_{\substack{j \neq i \\
j=1}}^{M} q_{j}^{L}(t)-\sum_{j=1}^{N} q_{j}^{F}(t+1) .
\end{aligned}
$$

The dynamics are affected by several factors. One of these factors is the number of leaders, while other factors include parameter values, such as the marginal costs $c$ and the initial conditions of the system at the start. The following three examples consider three sets of costs and they lead to different behaviors. For some parameter values, the players approach their steady states or stop producing (a zero steady state) after some time, as shown in Figure 1. For other parameter values, the behaviors of the players tend to limit cycles with period 2, as shown in Figure 2. We also observe cases where the dynamics of the players are chaotic, as shown in Figure 3.

\subsection{Leaders With Perfect Information}

Let us now consider the case of $M \geq 1$ leaders and $N \geq 1$ followers with perfect information for all of the leaders. The profit functions of an arbitrary leader or follower are

$$
\begin{aligned}
& \Pi_{i}^{L}(t+1)=\frac{A q_{i}^{L}(t+1)}{\sum_{j=1}^{M} q_{j}^{L}(t+1)+\sum_{j=1}^{N} q_{j}^{F}(t+1)}-c_{i}^{L} q_{i}^{L}(t+1), \\
& \Pi_{i}^{F}(t+1)=\frac{A q_{i}^{F}(t+1)}{q_{i}^{F}(t+1)+\sum_{j=1}^{M} q_{j}^{L}(t)+\sum_{\substack{j \neq i \\
j=1}}^{N} q_{j}^{F}(t)}-c_{i}^{F} q_{i}^{F}(t+1),
\end{aligned}
$$

respectively, where the superscript $L$ represents that the parameters and variables are related to the leaders while the superscript $F$ corresponds to the followers.

By working backward, the best responses of the followers can be determined first by solving $\frac{\partial \Pi_{i}^{F}}{\partial q_{i}^{F}}=0$. This results in

$$
q_{i}^{F}(t+1)=\sqrt{\frac{A}{c_{i}^{F}}\left(\sum_{j=1}^{M} q_{j}^{L}(t)+\sum_{\substack{j \neq i \\ j=1}}^{N} q_{j}^{F}(t)\right)}-\sum_{j=1}^{M} q_{j}^{L}(t)-\sum_{\substack{j \neq i \\ j=1}}^{N} q_{j}^{F}(t) .
$$


Then, the optimal responses of the leaders are obtained by solving $\frac{\partial \Pi_{i}^{L}}{\partial q_{i}^{L}}=0$, which results in

$$
q_{i}^{L}(t+1)=\sqrt{\frac{A}{c_{i}^{L}}\left(\sum_{\substack{j \neq i \\ j=1}}^{M} q_{j}^{L}(t+1)+\sum_{j=1}^{N} q_{j}^{F}(t+1)\right)}-\sum_{\substack{j \neq i \\ j=1}}^{M} q_{j}^{L}(t+1)-\sum_{j=1}^{N} q_{j}^{F}(t+1) .
$$

To compare the cases of perfect information and incomplete information, the following three examples use the same parameters as the examples with perfect information. For leaders with incomplete information, we found convergence to steady states, convergence to limit cycles, and then chaos. However, in the case of perfect information, the corresponding dynamics of these players are shown in Figures 4-6, and all cases yield convergence to equilibrium values in finite time. We observe a number of players exiting the game in this case. This suggests that perfect information regularizes the dynamics, resulting in rapid convergence to equilibrium values. While this is good for players facing low marginal costs, those players facing relatively higher marginal costs are forced out of the game.

By comparing the simulations, we find that the players which have leading outputs in three examples under incomplete information still have leading outputs under perfect information. However, some players facing higher costs which survive in the game with incomplete information are forced to quit the game in the case were leaders have perfect information. Unlike the systems with incomplete information in which various types of nonlinear dynamic behaviors can be found, the system with leaders of perfect information leads to steady state solutions in all the simulations we considered. Therefore, it would appear as if the perfect information for the leaders is stabilizing. However, the instability under incomplete information for the leaders can be beneficial for the players which are crowded out of the marker at steady state, as it allows those players to persist with positive quantities rather than leaving the market in finite time. For such players, non-equilibrium dynamics can actually be optimal. This concept was previously discussed for far simpler games (Papageorgiou, 2003), where it was suggested that chaotic or irregular dynamics may be beneficial under certain circumstances. See also Matsumoto (2006), where it was noted for Cournot games that players with low marginal costs prefer regular dynamics (e.g., equilibrium dynamics), while players facing higher marginal costs prefer non-equilibirum dynamics, such as chaos. What we have done here is noticed an analogous link between restrictive information structure and the prevalence of non-equilibrium dynamics which allow more of the players to persist in the dynamic game even if they face higher marginal costs.

\section{Multiple-Level Leader-Follower Games}

A more complicated case is the generalization of the multiple leader-follower game to a hierarchy with $L>1$ levels, where each level has $M_{l}$ players $(l=1,2, \ldots, L)$. One can view the game considered in Section 3 as a twolevel hierarchy leader-follower game. We shall assume that there is imperfect information available to a player about players in higher levels, but complete information about players in lower levels. Within the player's own level, there may or may not be complete information, depending on the information structure we choose within that level.

For the $i$ th player in the $l$ th level of the market (where $i=1,2, \ldots, M_{l}$ and $l=1,2, \ldots, L$ ), its profit function can be expressed as

$$
\Pi_{i}^{l}(t+1)=\frac{A q_{i}^{l}(t+1)}{q_{i}^{l}(t+1)+\sum_{k=1}^{l-1} \sum_{j=1}^{M_{k}} q_{j}^{k}(t)+\sum_{\substack{j \neq i \\ j=1}}^{M_{l}} q_{i}^{l}(t+\tau)+\sum_{k=l+1}^{L} \sum_{j=1}^{M_{k}} q_{j}^{k}(t+1)}-c_{i}^{l} q_{i}^{l}(t+1)
$$

Here, the parameter $\tau=0$ if the player has imperfect information about other players at its level, and $\tau=1$ if it has perfect information about other players at its level. Setting $\frac{d \Pi_{i}^{l}}{d q_{i}^{l}}=0$, we find the best response for this player, which is 
given by

$$
\begin{aligned}
q_{i}^{l}(t+1)= & \sqrt{\frac{A}{c_{i}^{l}}\left(\sum_{k=1}^{l-1} \sum_{j=1}^{M_{k}} q_{j}^{k}(t)+\sum_{\substack{j \neq i \\
j=1}}^{M_{l}} q_{i}^{l}(t+\tau)+\sum_{k=l+1}^{L} \sum_{j=1}^{M_{k}} q_{j}^{k}(t+1)\right)} \\
& -\sum_{k=1}^{l-1} \sum_{j=1}^{M_{k}} q_{j}^{k}(t)-\sum_{\substack{j \neq i \\
j=1}}^{M_{l}} q_{i}^{l}(t+\tau)-\sum_{k=l+1}^{L} \sum_{j=1}^{M_{k}} q_{j}^{k}(t+1) .
\end{aligned}
$$

Consider, for example, a three-level game under perfect information within each level $(\tau=1)$. In Figure 7 , we see that top-level leaders $(l=1)$ with lowest costs remain in the game and produce at stable equilibrium outputs. Others players quit the game. For other parameter values, we again observe convergence to steady states, rather than non-equilibrium dynamics, again due to the assumption of perfect information.

For our next example, we consider a four-level game under imperfect information $(\tau=0)$ within each level. Due to the imperfect information assumption, this game can exhibit a variety of non-equilibrium dynamics. Consider the case where each level has five players $\left(M_{l}=5\right.$ for $\left.l=1,2,3,4\right)$. For a variety of parameter values, such as those shown in Figures 8-9, chaotic dynamics can be observed. As was found in the previous section, such non-equilibrium dynamics allow for a larger number of players (albeit not all players in all cases) to remain in the game.

Equilibrium dynamics are still possible in this four-level game with imperfect equilibrium, but this naturally depends on the parameter values taken. To demonstrate this, we set all marginal costs to be equal, and consider the results in Figure 10. For this case, all players share the same equilibrium value, and will produce at this value for large enough time.

Bursting dynamics are observed in the chaotic regimes seen in the discrete time multi-level, multi-player leader follower game in Figure 8. Blue sky catastrophe is one example of such bursting chaos within the continuous time setting (Van Gorder, 2013). This suggests that, rather than exhibiting chaotic oscillations of similar but different amplitudes (as seen in the two-level games; see Figure 3), when there are many levels to the hierarchy, there may be intermittent regions of calm, with only small oscillations about some value of output, before much larger oscillations occur for another period of time, with the process repeating at irregular intervals. During the more "calm" periods, the leaders with more advantageous properties (lower marginal costs) will prosper, with other players facting reduced or zero quantities. Then, on the more "volatile" periods, drastic oscillations in the leaders with more advantageous properties will create an opening for the other players to resume production. In contrast, more standard chaotic oscillations are observed in Figure 9, and here players from all four levels are able to persist. Therefore, not only does chaos seem advantageous for the players which may face greater adversity under equilibrium dynamics, but the type of chaos observed also seems to influence the ability of such players to remain productive within the game.

\section{Discussion}

We study general multiple-player multiple-level dynamic discrete time leader-follower games in the vein of Cournot or Stackelberg games. Indeed, such games generalize two-player dynamic Stackelberg or Cournot duopoly games which have been considered recently in the discrete time setting. The most simple form of such a game is the two-level game, where there are a number of leaders and followers. However, one can generalize such games to any number of levels greater than two, with a given player acting as a leader toward players in a lower level, and a follower toward players in higher levels. We consider the case of either perfect or incomplete information, which in this context means that players either have complete information about other players within their level (perfect information) or lack information at the present timestep about other players within their level (incomplete information). Players always have perfect information about all players which are followers, and incomplete information about players which are leaders, relative to their level.

As we consider dynamic games, we observe a variety of behaviors in time, including convergence to steady state or equilibrium quantities, cycles or periodic oscillations, and chaotic dynamics. We find that the costs facing each player strongly influence the form of the long-time dynamics, as will the information structure (perfect or incomplete) selected. One interesting finding is that under perfect information players tend to quickly converge upon their respective equilibrium values, while incomplete information can result in loss of regularity and the emergence of periodic 
or chaotic dynamics. However, in cases where players may be pushed out of the game in the presence of high relative costs and perfect information, we find that non-equilibrium dynamics under incomplete information allow such players to retain positive production within the marker, hence they are able to remain in the game.

For some players, chaos may be beneficial, and this was previously shown for simpler games (Matsumoto, 2006; Papageorgiou, 2003). The inefficient player whose marginal cost is greater has higher long-run average profit than the expected profit earned in at the Cournot equilibrium in a unstable market while the efficient player with lower marginal costs has less long-run average profit (Matsumoto, 2006). As such, the former may prefer an unstable market. In our multi-level games, similar results were observed, with chaos permitting some, or even all, players to remain in the game. To a lesser extent, limit cycles also allowed some players to remain, when compared with equilibrium dynamics which often favored only the most efficient players. We also observed bursting type chaos in the four-level game, and when compared to regular chaos, it was possible for even more of the lower-tier players to remain competitive. Indeed, for regular chaos we observed that within a four-level game, some players within the top two levels remain in play, while for other parameters permitting bursting chaos, players from all four levels were able to remain in the game with positive output. Therefore, as non-equilibrium dynamics become increasingly disordered, less-efficient players appear to have more opportunity to persist through the game.

We also find that the information structure used by leaders in the two-level game, or players in a fixed level within the multi-level game, will strongly determine the dynamics. Recall that incorporating some types of delays can destabilizes the solutions of dynamical systems (Sipahi et al., 2011). As shown in Section 4, the incomplete information case is really equivalent to adding a delay to the resulting dynamics for each player, with the delay being that information about fellow players within a fixed level is only available at previous timesteps rather than the current time. This can result in a destabilization of the dynamics, which in turn brings about non-equilibrium dynamics such as limit cycles or chaos (depending on the parameter regime). In contrast, for the case of perfect information, players within a fixed level have complete information about one another up to the current time. For perfect information, we observe stable equilibrium dynamics, and less-efficient players are often crowded out of the game for small values of time. However, as mentioned above, the non-equilibrium dynamics can be of use to players which are less efficient. This means that efficient players (those facing lower marginal costs) will prefer perfect information about competitors within the same level in the hierarchy, while less-efficient players may prefer incomplete information, as the resulting loss of equilibrium for the more efficient leaders gives these other players the opportunity to remain productive in the game.

[1] Agiza, H N, Elsadany, A A (2003) Nonlinear dynamics in the Cournot duopoly game with heterogeneous players. Physica A 320: 512-524

[2] Agliari, A, Gardini, L, Puu, T (2000) The dynamics of a triopoly Cournot game. Chaos, Solitons \& Fractals 11(15): 2531-2560

[3] Ahmed, E, Hegazi, A S (2006) On dynamical multi-team and signaling games. Applied Mathematics and Computation 172(1): 524-530.

[4] Askar, S S, Alnowibet, K (2016) Cooperation versus noncooperation: Cournot duopolistic game based on delay and time-dependent parameters. Chaos, Solitons \& Fractals 91: 580-584

[5] Basar, T, Olsder, G J (1980) Team-optimal closed-loop Stackelberg strategies in hierarchical control problems. Automatica 16: 409-414

[6] Cruz, J B (1976) Stackelberg strategies for multilevel systems. Directions in Large-Scale Systems, p. 139-147, Springer US

[7] Cruz, J (1978) Leader-follower strategies for multilevel systems. IEEE Transactions on Automatic Control 23: 244-255

[8] D'Amato, E, Daniele, E, Mallozzi, L, Petrone, G, Tancredi, S (2012) A Hierarchical MultiModal Hybrid Stackelberg-Nash GA for a Leader with Multiple Followers Game. In Dynamics of Information Systems: Mathematical Foundations (pp. 267-280). Springer New York.

[9] D’Orsogna, M R, Perc, M (2015) Statistical physics of crime: A review. Physics of Life Reviews 12: 1-21.

[10] Elsadany, A A, Agiza, H N, Elabbasy, E M (2013) Complex dynamics and chaos control of heterogeneous quadropoly game. Applied Mathematics and Computation 219(24): 11110-11118

[11] Gardner, B, Cruz, J (1978) Feedback Stackelberg strategy for M-level hierarchical games. IEEE Transactions on Automatic Control 23: 489-491

[12] Guruacharya, S, Niyato, D, Kim, D I, Hossain, E (2013) Hierarchical competition for downlink power allocation in OFDMA femtocell networks. IEEE Transactions on Wireless Communications 12(4): 1543-1553

[13] Helbing, D, Brockmann, D, Chadefaux, T., Donnay, K, Blanke, U, Woolley-Meza, O, Moussaid, M, Johansson, A, Krause, J, Schutte, S, Perc, M 2015 Saving human lives: what complexity science and information systems can contribute. Journal of Statistical Physics 158(3): 735-781.

[14] Li, M, Cruz, J B, Simaan, M A (2002) An approach to discrete-time incentive feedback Stackelberg games. IEEE Transactions on Systems, Man, and Cybernetics-Part A: Systems and Humans 32(4): 472-481

[15] Matsumoto, A (2006) Controlling the Cournot-Nash Chaos. Journal of Optimisation Theory and Application 128(2): $379-392$

[16] Nie, P Y (2007) Discrete time dynamic multi-leader-follower games with feedback perfect information. International Journal of Systems Science 38(3): 247-255

[17] Pan, L, Yong, J (1991) A differential game with multi-level of hierarchy. Journal of Mathematical Analysis and Applications 161(2): $522-544$

[18] Pang, J S, Fukushima, M (2005) Quasi-variational inequalities, generalized Nash equilibria, and multi-leader-follower games. Computational Management Science 2(1): 21-56 
[19] Papageorgiou, M (2003) Chaos May Be an Optimal Plan. Journal of Optimization Theory and Applications 2(119): 387-393.

[20] Perc, M (2016) Phase transitions in models of human cooperation. Physics Letters A 380: 2803-2808.

[21] Peng, Y, Lu, Q (2015) Complex dynamics analysis for a duopoly Stackelberg game model with bounded rationality. Applied Mathematics and Computation, 271: 259-268

[22] Peng, Y, Lu, Q, Xiao, Y (2016) A dynamic Stackelberg duopoly model with different strategies. Chaos, Solitons \& Fractals 85: 128-134

[23] Price, M E, Van Vugt, M (2014) The evolution of leader-follower reciprocity: the theory of service-for-prestige. Frontiers in Human Neuroscience 8: 363

[24] Puu, T (1991a) Chaos in duopoly pricing. Chaos, Solitons \& Fractals 1(6): 573-581

[25] Puu, T (1991b) Nonlinear economic dynamics. In Nonlinear Economic Dynamics (pp. 1-7). Springer Berlin Heidelberg

[26] Puu, T (1998) The chaotic duopolists revisited. Journal of Economic Behavior \& Organization 33(3): 385-394

[27] Puu, T (2008) On the stability of Cournot equilibrium when the number of competitors increases. Journal of Economic Behavior \& Organization 66(3): 445-456

[28] Rassenti, S, Reynolds, S S, Smith, V L, Szidarovszky, F 2000 Adaptation and convergence of behavior in repeated experimental Cournot games. Journal of Economic Behavior \& Organization 41(2): 117-146.

[29] Shen, H, Basar, T (2004) Differentiated Internet pricing using a hierarchical network game model. In: Proceedings of the 2004 American Control Conference 3: 2322-2327

[30] Sherali, H D, Soyster, A L, Murphy, F H (1983) Stackelberg-Nash-Cournot equilibria: characterizations and computations. Operations Research 31(2): 253-276

[31] Sherali, H D (1984) A multiple leader Stackelberg model and analysis. Operations Research 32(2): 390-404

[32] Sipahi, R, Niculescu, S I, Abdallah, C T, Michiels, W, Gu, K (2011) Stability and stabilization of systems with time delay. IEEE Control Systems 31(1): 38-65

[33] Snyder, B C, Van Gorder, R A, Vajravelu, K (2013) Continuous-time dynamic games for the Cournot adjustment process for competing oligopolists. Applied Mathematics and Computation 219: 6400-6409

[34] Tramontana, F, Gardini, L, Puu, T (2011) Mathematical properties of a discontinuous Cournot-Stackelberg model. Chaos, Solutions \& Fractals 44: $58-70$

[35] Tzafestas, S G (1980) Multilevel stackelberg control of distributed-parameter systems. In Analysis and Optimization of Systems (pp. $462-$ 485). Springer Berlin Heidelberg.

[36] Valletti, T M 2000 Minimum quality standards under Cournot competition. Journal of Regulatory Economics 18(3): 235-245.

[37] Van Gorder, R A, Caputo, M R (2010) Envelope theorems for locally differentiable open-loop Stackelberg equilibria of finite horizon differential games. Journal of Economic Dynamics and Control 34(6): 1123-1139

[38] Van Gorder, R A (2013) Triple mode alignment in a canonical model of the blue-sky catastrophe. Nonlinear Dynamics 73: $397-403$.

[39] Von Stackelberg, H (1934) Marktform und gleichgewicht. J. springer. Julius Springer, Vienna.

[40] Xu, Y, Lui, J C, Chiu, D M (2010) On oligopoly spectrum allocation game in cognitive radio networks with capacity constraints. Computer Networks 54(6): 925-943.

[41] Wang, Z, Bauch, C T, Bhattacharyya, S, d'Onofrio, A, Manfredi, P, Perc, M, Perra, N, Salathé, M and Zhao, D (2016) Statistical physics of vaccination. Physics Reports 664: 1-113.

[42] Wernerfelt, B 1989 Tacit collusion in differentiated Cournot games. Economics Letters 29(4): 303-306. 


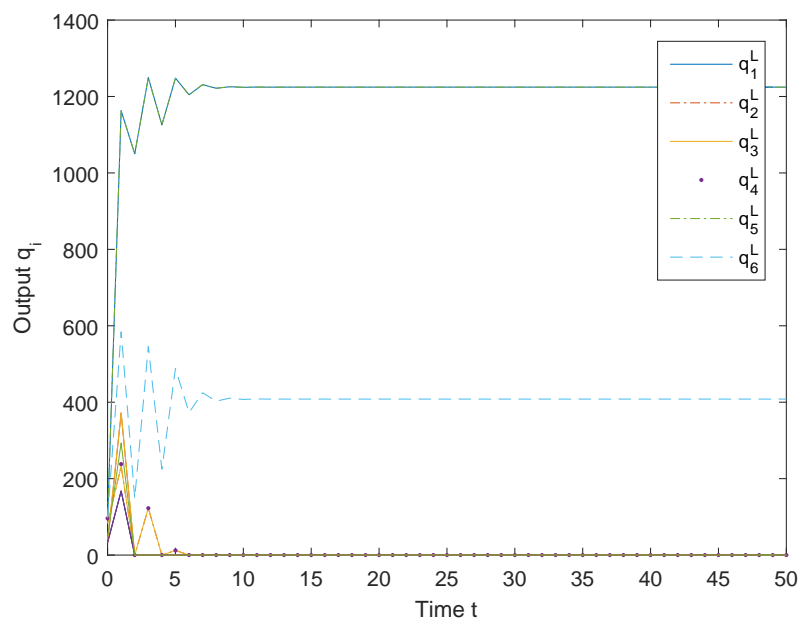

Figure 1: Output quantities for each player in the game with $M=6$ leaders and $N=6$ followers under incomplete information for the leaders. Parameter values are $A=10000, c^{L}=[2 ; 4 ; 4 ; 4 ; 2 ; 3], c^{F}=[9 ; 9 ; 6 ; 6 ; 9 ; 7], \mathbf{q}^{L}(0)=[93 ; 84 ; 83 ; 96 ; 97 ; 99]$, and $\mathbf{q}^{F}(0)=[32 ; 33 ; 32 ; 40 ; 34 ; 48]$. The green line and the dark blue line (which is covered by the green line as their outputs are the same after first few steps) represent the players with cost 2 and their outputs are the highest. Many other players, including come leaders, quit the game after some time since their costs are too high to compete with the leaders with low costs.

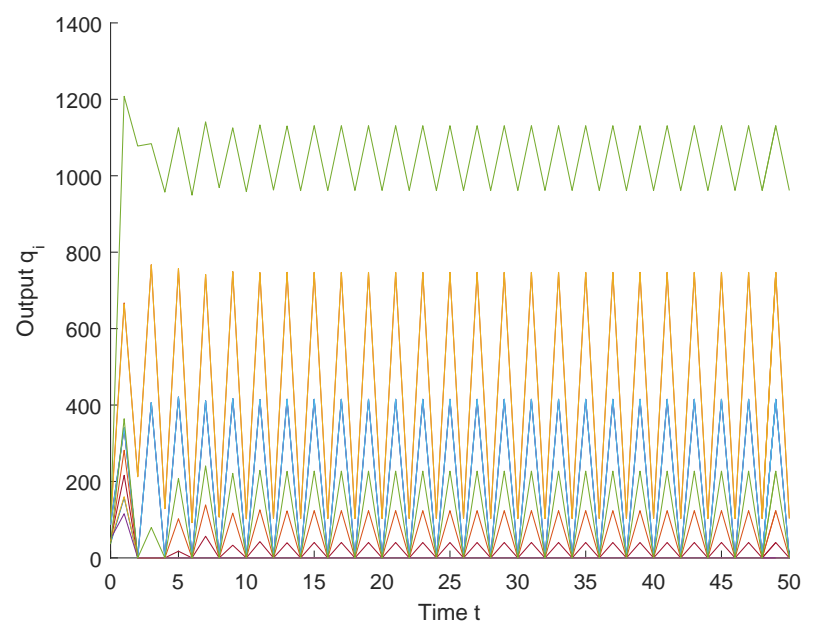

Figure 2: Output quantities for each player in the game with $M=6$ leaders and $N=6$ followers under incomplete information for the leaders. Parameter values are $A=10000, c^{L}=[4 ; 3 ; 3 ; 4 ; 2 ; 4], c^{F}=[8 ; 9 ; 7 ; 9 ; 10 ; 6], \mathbf{q}^{L}(0)=[91 ; 100 ; 91 ; 86 ; 93 ; 87]$, and $\mathbf{q}^{F}(0)=[45 ; 38 ; 40 ; 44 ; 50 ; 36]$. The players with lower marginal cost produce at higher outputs. Some of the players with relatively high costs will exit the game. In this example we observe the emergence of limit cycles after several timesteps. 


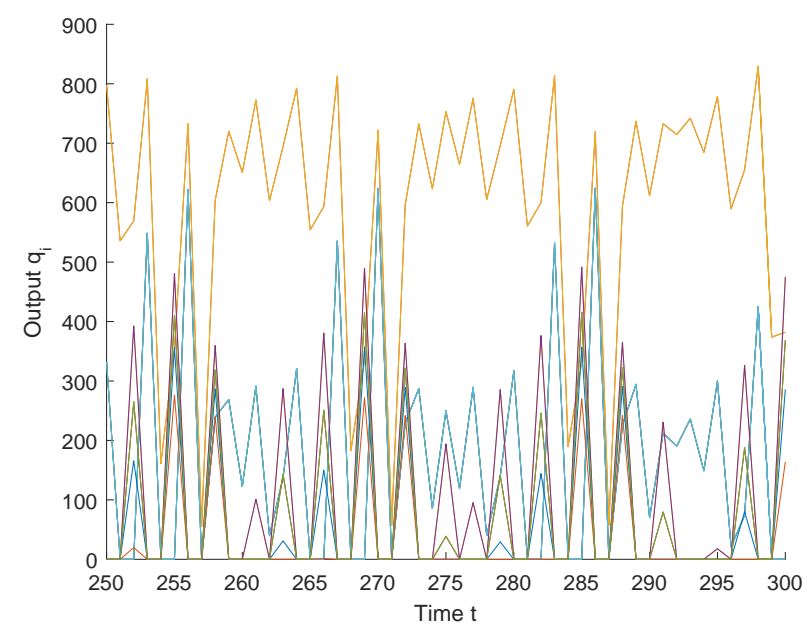

Figure 3: Output quantities for each player in the game with $M=6$ leaders and $N=6$ followers under incomplete information for the leaders. Parameter values are $A=10000, c^{L}=[4 ; 3 ; 3 ; 4 ; 4 ; 4], c^{F}=[6 ; 7 ; 9 ; 5 ; 5 ; 6], \mathbf{q}^{L}(0)=[91 ; 100 ; 94 ; 86 ; 86 ; 97]$, and $\mathbf{q}^{F}(0)=[49 ; 43 ; 35 ; 31 ; 37 ; 42]$. The dynamics observed are non-equilibrium, irregular (non-repeating) oscillations - rather than limit cycles. Having run this simulation for many iterations and not observed a repeat or signs of quasi-periodicity, we conclude these dynamics are chaotic. In this example, all the players produce at some non-zero level for some non-bounded values of time (if not all time), so there is no player quitting the game.

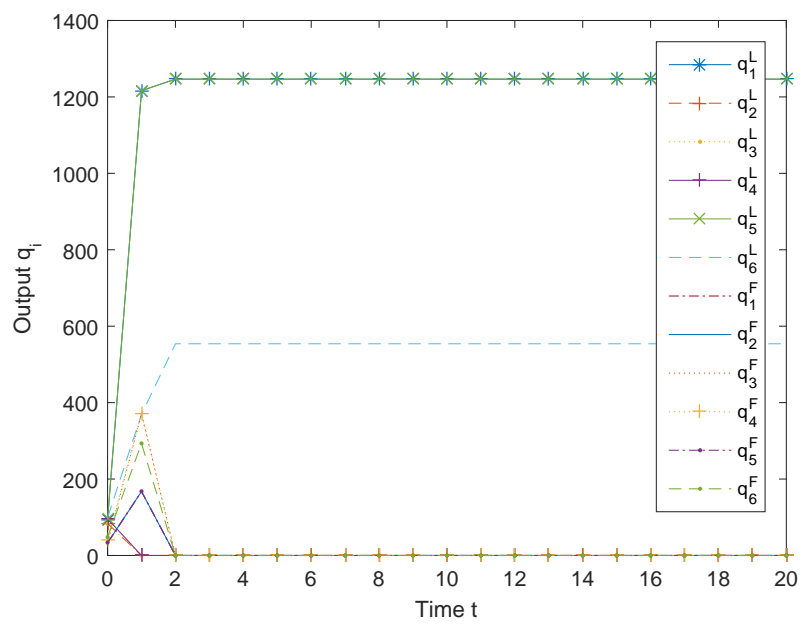

Figure 4: Output quantities for each player in the game with $M=6$ leaders and $N=6$ followers under perfect information for the leaders. Parameter values are $A=10000, c^{L}=[2 ; 4 ; 4 ; 4 ; 2 ; 3], c^{F}=[9 ; 9 ; 6 ; 6 ; 9 ; 7], \mathbf{q}^{L}(0)=[93 ; 84 ; 83 ; 96 ; 97 ; 99]$, and $\mathbf{q}^{F}(0)=[32 ; 33 ; 32 ; 40 ; 34 ; 48]$. These equilibrium values are the same as the example with incomplete information shown in Figure 1, although the very small time dynamics differ. 


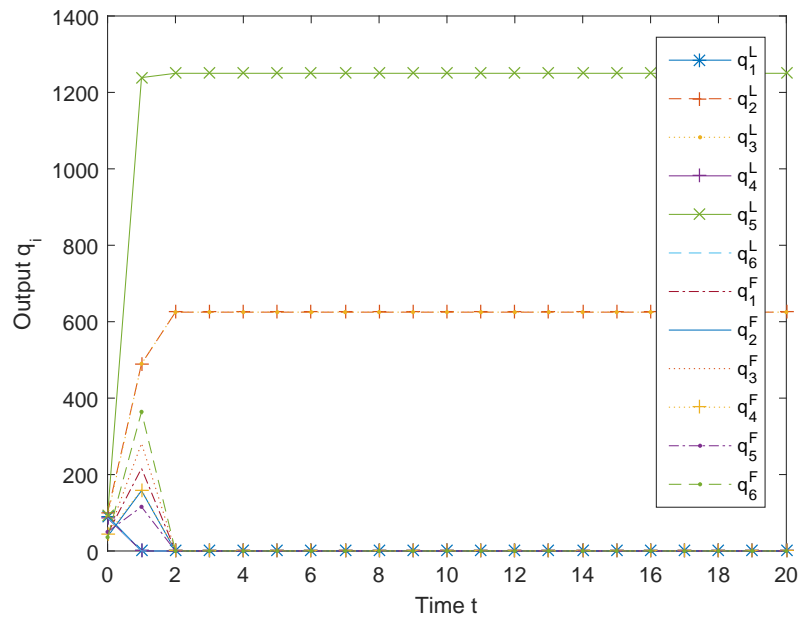

Figure 5: Output quantities for each player in the game with $M=6$ leaders and $N=6$ followers under perfect information for the leaders. Parameter values are $A=10000, c^{L}=[4 ; 3 ; 3 ; 4 ; 2 ; 4], c^{F}=[8 ; 9 ; 7 ; 9 ; 10 ; 6], \mathbf{q}^{L}=[91 ; 100 ; 91 ; 86 ; 93 ; 87]$, and $\mathbf{q}^{F}=[45 ; 38 ; 40 ; 44 ; 50 ; 36]$. In this case we observe convergence to equilibrium values, rather than the limit cycles observed for the same parameter values under incomplete information in Figure 2. Most players stop producing after time $t=2$.

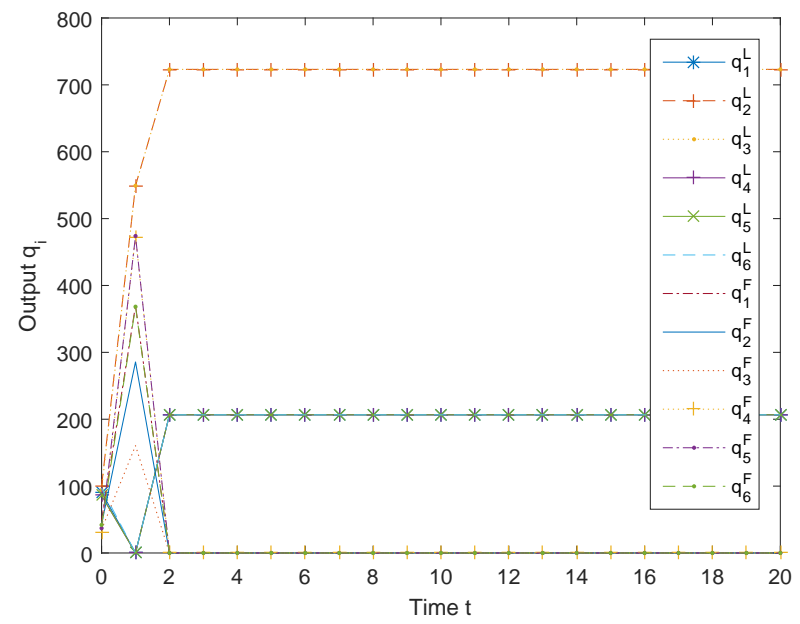

Figure 6: Output quantities for each player in the game with $M=6$ leaders and $N=6$ followers under perfect information for the leaders. Parameter values are $A=10000, c^{L}=[4 ; 3 ; 3 ; 4 ; 4 ; 4], c^{F}=[6 ; 7 ; 9 ; 5 ; 5 ; 6], \mathbf{q}^{L}(0)=[91 ; 100 ; 94 ; 86 ; 86 ; 97]$, and $\mathbf{q}^{F}(0)=[49 ; 43 ; 35 ; 31 ; 37 ; 42]$. In this case we observe convergence to equilibrium values, rather than the chaos observed for the same parameter values under incomplete information in Figure 3. Most players stop producing after time $t=2$. 


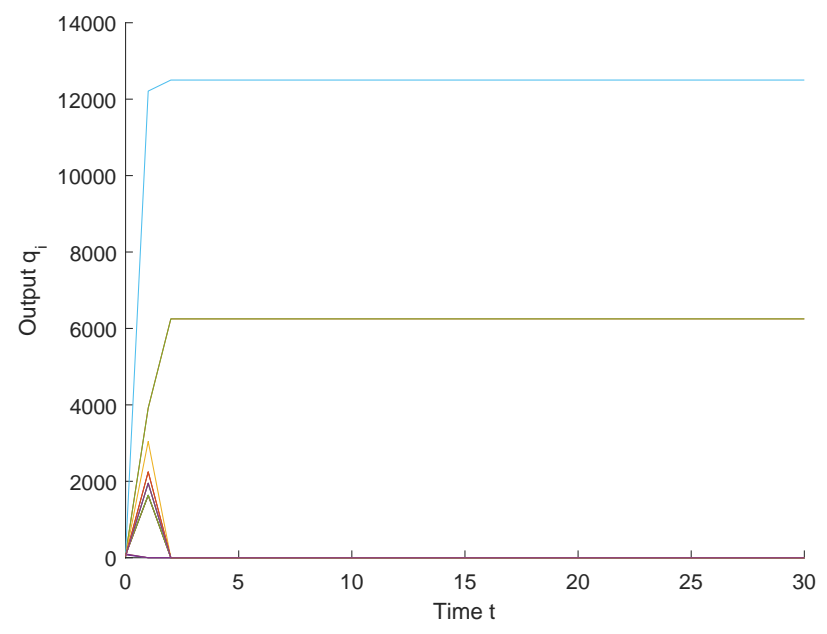

Figure 7: Output quantities for players in a three-level game $(L=3)$ where each level has $M_{l}=6$ players $(l=1,2,3)$, under the assumption of perfect information. Parameter values are $A=100000, c^{1}=[4 ; 3 ; 4 ; 4 ; 3 ; 2], c^{2}=[6 ; 6 ; 6 ; 5 ; 8 ; 6], c^{3}=[12 ; 10 ; 12 ; 10 ; 12 ; 12], \mathbf{q}^{1}(0)=$ $[87 ; 84 ; 96 ; 99 ; 86 ; 94], \mathbf{q}^{2}(0)=[63 ; 75 ; 73 ; 55 ; 76 ; 80]$, and $\mathbf{q}^{3}(0)=[40 ; 48 ; 42 ; 33 ; 34 ; 38]$. For this parameter set, may players are forced out of the game due to relatively higher marginal costs. Those players remaining in the game rapidly converge to their equilibrium values. It is the top-level leaders which persist in this game.

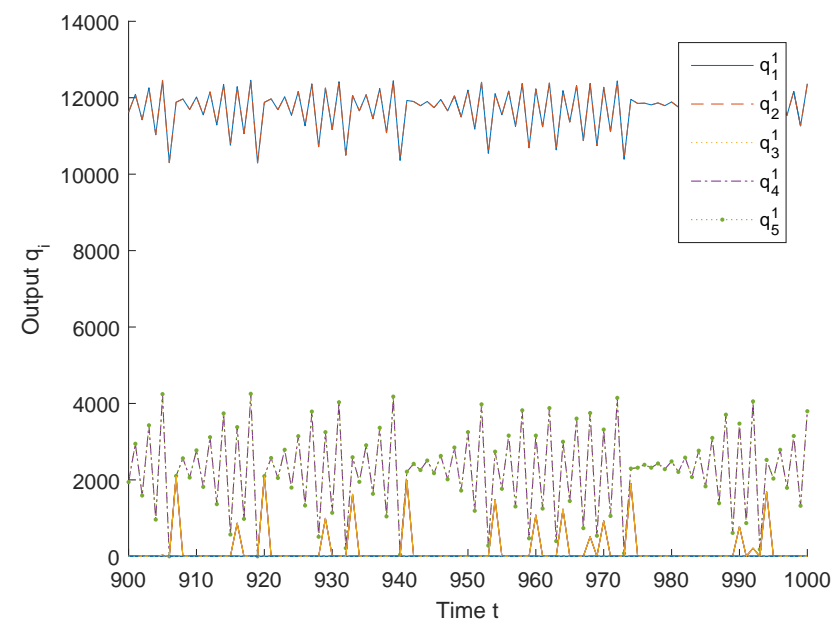

Figure 8: Output quantities for players in a four-level game $(L=4)$ where each level has $M_{l}=5$ players $(l=1,2,3,4)$, under the assumption of imperfect information. Parameter values are $A=100000, c^{1}=[2 ; 2 ; 3 ; 3 ; 3] c^{2}=[4 ; 4 ; 5 ; 4 ; 4], c^{3}=[6 ; 6 ; 6 ; 7 ; 6], c^{4}=[10 ; 10 ; 10 ; 10 ; 10]$, $\mathbf{q}^{1}(0)=[176 ; 166 ; 171 ; 153 ; 195], \mathbf{q}^{2}(0)=[103 ; 122 ; 142 ; 131 ; 141], \mathbf{q}^{3}(0)=[95 ; 97 ; 59 ; 63 ; 80]$ and $\mathbf{q}^{4}(0)=[35 ; 38 ; 45 ; 26 ; 34]$. We observe chaos in the four-level game for these parameter values. As found previously, the chaotic dynamics allow for a larger number of players to remain in the game. While some of the players are forced out of the game, members of the top two levels remain if they face low enough costs. 


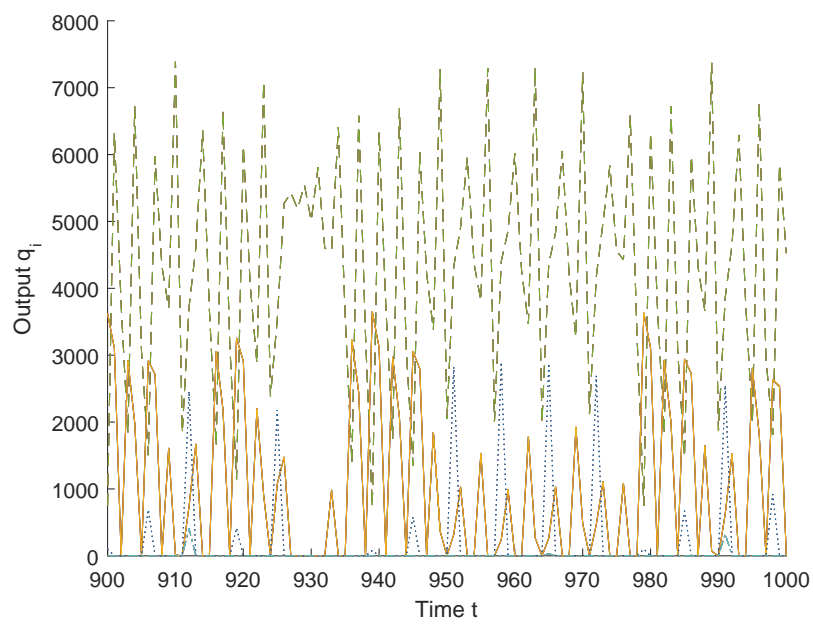

Figure 9: Output quantities for players in a four-level game $(L=4)$ where each level has $M_{l}=5$ players $(l=1,2,3,4)$, under the assumption of imperfect information. Parameter values are $A=100000, c_{i}^{1}=3, c_{i}^{2}=4, c_{i}^{3}=6, c_{i}^{4}=10$ (for $\left.i=1,2,3,4,5\right), \mathbf{q}^{1}(0)=[176 ; 166 ; 171 ; 153 ; 195]$, $\mathbf{q}^{2}(0)=[103 ; 122 ; 142 ; 131 ; 141], \mathbf{q}^{3}(0)=[95 ; 97 ; 59 ; 63 ; 80]$ and $\mathbf{q}^{4}(0)=[35 ; 38 ; 45 ; 26 ; 34]$. We again observe chaotic dynamics, although for these parameter values, players from all four levels persist in the game and produce positive output.

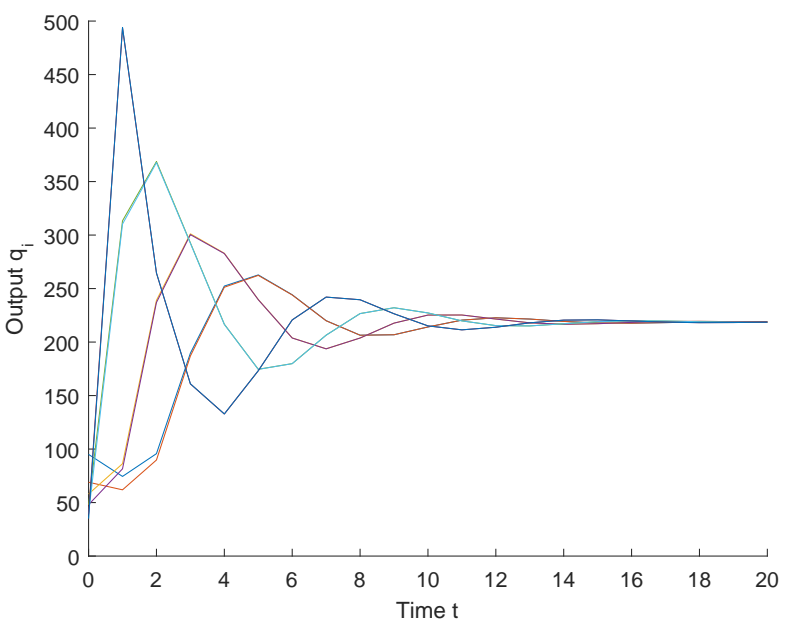

Figure 10: Output quantities for players in a four-level game $(L=4)$ where each level has $M_{l}=5$ players $(l=1,2,3,4)$, under the assumption of imperfect information. Parameter values are $A=100000, c_{i}^{l}=5$ for all $l=1,2,3,4$ and $i=1,2,3,4,5, \mathbf{q}^{1}(0)=[95 ; 69], \mathbf{q}^{2}(0)=[58 ; 48]$, $\mathbf{q}^{3}(0)=[48 ; 40]$ and $\mathbf{q}^{4}=[39 ; 35]$. Here the players all remain in the game, and produce at their equilibrium values for large enough time. 